\title{
Inhibition of colon tumor growth by IL-15 immunogene therapy
}

\author{
XIANGHUI HE ${ }^{1}$, WEIDONG LI ${ }^{1}$, YIFENG WANG ${ }^{2}$, LINAN HOU $^{3}$ and LIWEI ZHU ${ }^{1}$ \\ ${ }^{1}$ Department of General Surgery; ${ }^{2}$ Intensive Care Unit, Tianjin General Surgery Institute, Tianjin Medical University \\ General Hospital, Tianjin 300052; ${ }^{3}$ Department of Surgery, Tianjin Wuqing Hospital, Tianjin 300170, P.R. China
}

Received July 13, 2011; Accepted September 23, 2011

DOI: 10.3892/mmr.2011.601

\begin{abstract}
Interleukin-15 is a pleiotropic cytokine that has potential for cancer immunegene therapy. We previously reported the construction and characterization of IL-15 overexpression vectors pHi2-IL15-CMV-tat (L1) and pHi2-spIL15-CMV-tat (L3), as well as carcinoembryonic antigen promoter-amplified IL-15 expression plasmid vectors pHi2-IL15-CEA-tat (L2) and pHi2-spIL15-CEA-tat (L4). In the current study, we evaluated the expression and therapeutic efficacy of these vectors using a mouse colon carcinoma model. Plasmid vectors were transfected into SW480 and MCF-7 cells, and IL-15 overexpression was confirmed. IL-15 expressed by transfected tumor cells stimulated spleen cell proliferation in vitro. Intraperitoneal injection of plasmid pHi2-spIL15-CMV-tat (L3) into mice resulted in transgene expression by peritoneal mesothelial cells, inhibited CT-26 tumor growth and prolonged the survival of tumor-bearing mice. In addition, the in vivo transfection of plasmid pHi2-spIL15-CMV-tat (L3) via electroporation slowed the tumor formation of subcutaneously inoculated CT-26 cells. These data suggest that IL-15 overexpression achieves therapeutic effects in a mouse cancer model and that these gene transfer approaches should be further evaluated for use in the treatment of human cancers.
\end{abstract}

\section{Introduction}

Immunotherapy has emerged as a promising approach for cancer treatment. Cytokine-based immune gene therapy, aimed to boost the anti-tumor immune responses via cytokine gene transfer, has been rigorously investigated. Numerous cytokines have been tested in preclinical settings and in phase I/II gene therapy clinical trials, including granulocytemacrophage colony-stimulating factor (GM-CSF), tumor necrosis factor- $\alpha$ (TNF- $\alpha)$, IFN- $\gamma$, interleukin-2 and interleukin-12 (1,2). Currently, interleukin-15 (IL-15) is attracting

Correspondence to: Dr Xianghui He, Department of General Surgery, Tianjin General Surgery Institute, Tianjin Medical University General Hospital, No. 154 Anshan Rd., Tianjin 300052, P.R. China

E-mail: humphreyhe@163.com

Key words: cancer, gene therapy, interleukin-15 attention for its use in cancer immune gene therapy due to its specific activities on both innate and adaptive immune cells.

IL-15 is a pleiotropic cytokine that promotes the expansion, activation and maintenance of natural killer $(\mathrm{NK})$ cells and $\mathrm{CD}^{+} \mathrm{T}$ cells, as well as stimulating the function of neutrophils, macrophages and dendritic cells (3). IL-15 is a potent inducer of CC-, CXC- and C-type chemokine expression in $\mathrm{T}$ lymphocytes. In contrast to IL-2, IL-15 inhibits antigen-induced cell death, and previous data have shown that IL-15 is capable of reversing T-cell anergy (4). Therefore, IL-15 is a promising cytokine for cancer immunotherapy. However, IL-15 signaling occurs through a complicated trans-presentation mechanism by binding to its high affinity receptor IL-15R $\alpha$ (5), which limits the use of recombinant IL-15 in cancer therapy. Expressing IL-15 in vivo via a genetic approach may therefore be a more effective strategy. In a previous study (6) we constructed the IL-15 overexpression plasmid vector, pHi2-spIL15-CMV-tat, as well as the carcinoembryonic antigen (CEA) promoter-controlled IL-15 overexpression plasmid vector, pHi2-spIL15-CEA-tat. In both of these vectors, the HIV2 promoter directs IL-15 expression and a CMV or CEA promoter drives the expression of the transcriptional transactivator, tat, to enhance the efficacy of the HIV2 promoter. The CEA-specific positively controlled IL-15 overexpression plasmid led to a significant amount of IL-15 production in CEA-positive human colon carcinoma SW480 cells (6). In the present study, we further evaluated the expression and therapeutic efficacy of these vectors in vitro and in vivo using a clinically relevant gene transfer approach for IL-15 gene therapy in a mouse model. The data revealed that IL-15 expressed by transfected tumor cells stimulated spleen cell proliferation in vitro, and that IL-15 gene transfer enhanced NK cell activity. Intraperitoneal injection of the pHi2-spIL15-CMV-tat plasmid resulted in transgene expression by peritoneal mesothelial cells and inhibited mouse colon carcinoma CT-26 tumor growth in vivo. In addition, transfection of plasmid pHi2-spIL15-CMV-tat via electroporation slowed subcutaneously inoculated CT-26 tumor formation.

\section{Materials and methods}

Animal and cell lines. BALB/c mice (aged 6-12 weeks) were purchased from the animal center of the Institute of Radiation Medicine, Chinese Academy of Medical Sciences (Tianjin, China) and were maintained in the animal facility at the 
Tianjin Medical University General Hospital. Human colon carcinoma SW480 cells, human breast carcinoma MCF-7 cells and mouse colon carcinoma CT-26 cells were obtained from the American Type Culture Collection (Manassas, VA, USA). Cells were maintained in RPMI-1640 supplemented with $10 \%$ fetal bovine serum (Invitrogen, Beijing, China), $2 \mathrm{mM}$ glutamine, $1 \mathrm{mM}$ pyruvate, $50 \mu \mathrm{M}$ 2-mercaptoethanol, penicillin $(200 \mathrm{U} / \mathrm{ml})$ and streptomycin $(200 \mu \mathrm{g} / \mathrm{ml})$ at $37^{\circ} \mathrm{C}$ in a $5 \%$ $\mathrm{CO}_{2} / 95 \%$ air atmosphere.

Genetic constructs. The IL-15 expression plasmid vectors used in this study were pHi2-IL15-CMV-tat (L1), pHi2-IL15-CEA-tat (L2), pHi2-spIL15-CMV-tat (L3) and pHi2-spIL15-CEA-tat (L4). The construction and characterization of these vectors has previously been described (6). Briefly, IL-15 expression was driven by the HIV2 promoter in all these vectors and the activity of the HIV2 promoter was amplified by the expression of tat driven by the CMV promoter (L1 and L3) or the CEA promoter (L2 and L4). In plasmids pHi2-spIL15-CMV-tat (L3) and pHi2-spIL15-CEA-tat (L4), high levels of IL-15 expression were achieved by replacing the IL-15 signal sequence with the IL-2 signal sequence. In addition, pHi2-EGFP-CMV-tat (L6) vectors, in which the gene encoding enhanced green fluorescence protein (EGFP) was cloned into the same backbone plasmid as L1 behind the HIV2 promoter, were used to evaluate transfection efficiency. Backbone vector pHi2-MCS-CMV-tat was used as a control and was generously provided by Dr Harris at the University of Arizona (AZ, USA).

Cell transfection. Tumor cells were transfected with plasmid DNA by electroporation. Briefly, cells were plated at a concentration of $5 \times 10^{5}$ cells/well in $2 \mathrm{ml}$ medium in 6 -well plates and grown to $70-80 \%$ confluence. Just prior to transfection, the culture medium was removed and cells were washed with phosphate-buffered saline (PBS). A $20 \mu \mathrm{g}$ plasmid DNA diluted in $100 \mu 1$ OptiMEM (Gibco, Rockville, MD, USA) medium was added to each well. Pulses were applied to the tumor cells through the petri dish electrode using a power supply device (BTX ECM 830, Genetronic, CA, USA). For MCF-7 cells, the parameters were $140 \mathrm{~V} / \mathrm{cm}$, six 20-msec pulses. For SW480 cells, the parameters were $200 \mathrm{~V} / \mathrm{cm}$, six 20-msec pulses. Following electroporation, fresh RPMI was added to the well for a total volume of $2 \mathrm{ml}$. Cells were cultured for $24 \mathrm{~h}$, after which time the culture supernatant was collected.

Enzyme-linked immunosorbent assay (ELISA) for IL-15 production. Following $24 \mathrm{~h}$ of transfection, culture supernatants were collected and tested for IL-15 expression using ELISA according to the manufacturer's instructions (R\&D Systems, MN, USA). Briefly, a 96-well plate was coated with anti-mouse IL-15 antibody and blocked with bovine serum albumin-containing buffer. After washing the cells, a standardized IL-15 solution and the cell culture supernatants were added to the wells. Following $2 \mathrm{~h}$ of incubation, the plate was washed, and the biotin-labeled detection antibody and avidinhorseradish peroxidase (HRP) were added. Following an additional 1-h incubation and washing, the substrate solution was added and absorbance was detected at $450 \mathrm{~nm}$.
Cell proliferation assay. Spleen cells were harvested from BALB/c mice and were cultured at a concentration of $4 \times 10^{6} / \mathrm{ml}$ in RPMI for $48 \mathrm{~h}$ with $100 \mu \mathrm{l}$ culture supernatants from transfected cells. A total of $20 \mu \mathrm{l}$ of methyl thiazolyl tetrazolium (MTT) $(5 \mathrm{mg} / \mathrm{ml})$ was then added to the wells and the assay plates were incubated for an additional $4 \mathrm{~h}$. The supernatants were discarded and $150 \mu \mathrm{l}$ of DMSO was added to each well. After the crystals dissolved, the $490 \mathrm{~nm}$ absorbance was detected using a microplate reader.

NK cell cytotoxicity assay. BALB/c mice were injected intraperitoneally with IL-15-expressing plasmid or backbone plasmid at days 1,4 and 7. A group of mice were administered PBS solution as a control. Mice were sacrificed at day 10 and their spleens were harvested. NK cells were isolated by negative selection using MACS NK Cell Isolation Kit (Miltenyi Biotec Inc., CA, USA) according to the manufacturer's instructions. Briefly, spleen cells were treated with biotin-antibody cocktail and then mixed with anti-biotin microbeads. After washing with buffer, the cell suspension was applied onto the column placed in the magnetic field in a MidiMACS Separator (Miltenyi Biotec). Unlabeled cells that passed through the column were collected as effector cells. The Yac-1 cells were used as target cells. Cytotoxicity assays were performed using a CytoTox96 non-radioactive cytotoxicity assay kit (Promega, Madison, WI, USA). Briefly, a constant number of target cells and various numbers of effector cells were co-cultured in $100 \mu \mathrm{l}$ of medium in a round-bottomed 96-well plate for 12 h. A total of $50 \mu \mathrm{l}$ of supernatant was harvested and lactate dehydrogenase (LDH) in the supernatant was measured using a 30-min coupled enzymatic assay. Absorbance data at $490 \mathrm{~nm}$ were collected using a microplate reader.

In vivo animal fluorescence optical imaging. In vivo animal fluorescence optical imaging was performed using the Xenogen system (Xenogen, Alameda, CA, USA). Twenty four hours prior to imaging, each mouse was administered an intraperitoneal injection of $200 \mu \mathrm{l}$ of formulation containing $50 \mu \mathrm{g}$ plasmid DNA or $200 \mu \mathrm{l}$ PBS. Just prior to imaging, the mouse was sacrificed and its abdomen opened, and it was placed in the light-tight chamber. Fluorescent images and bright field photographs were captured for each imaging time. The merged bright field photographs and fluorescent images were generated and analyzed with the Igor (WaveMetrics, Lake Oswego, OR, USA) and IVIS Living Image (Xenogen, Alameda) software packages.

In vivo animal study. Mice were inoculated intraperitoneally with $1 \times 10^{7}$ CT-26 cells, treated by abdominal injections of PBS, plasmid pHi2-MCS-CMV-tat or pHi2-spIL15-CMV-tat on days 3, 5 and 7, and were monitored for survival. For intra-tumor gene transfer, BALB/c mice were subcutaneously injected with $5 \times 10^{6} \mathrm{CT}-26$ cells. When the tumors reached 6-8 $\mathrm{mm}$ in diameter at day 7 , the animals were anesthetized and injected with purified plasmids formulated at 0.1 M PBS. A formulation containing $50 \mu \mathrm{g}$ DNA was injected directly into each tumor for the experimental group while the control group received an injection of $50 \mu \mathrm{g}$ naked plasmid. For electroporation following DNA injection, the two heads of a caliper electrode were positioned on opposite sides of the tumor with 
A

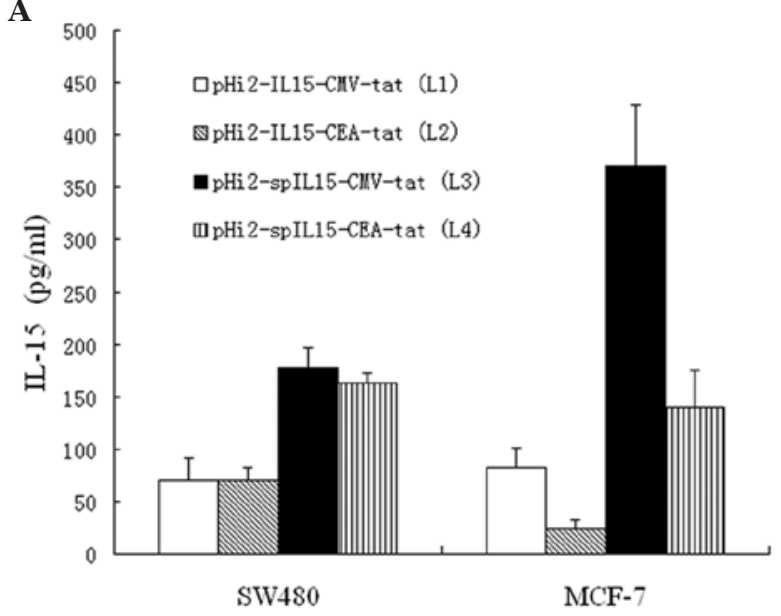

B

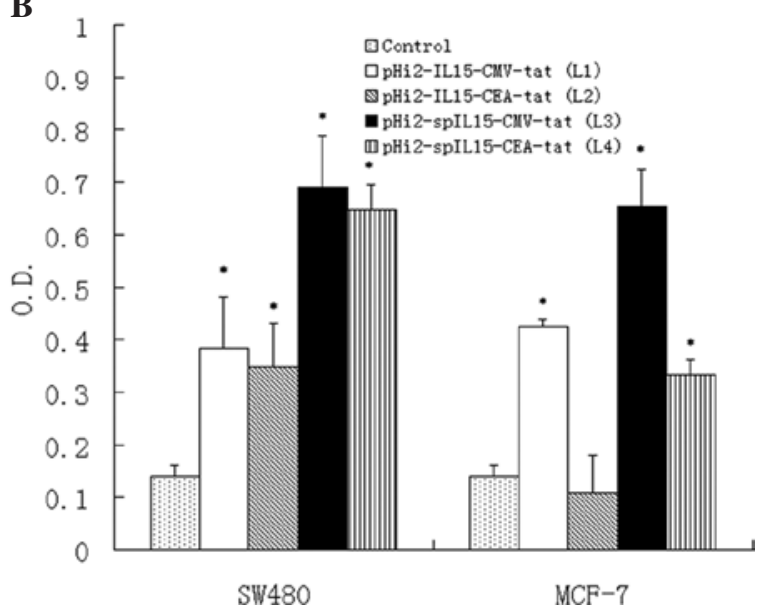

Figure 1. IL-15 expressed by transfected tumor cells stimulated spleen cell proliferation in vitro. (A) SW480 and MCF-7 cells were transfected with the IL-15 expression plasmids pHi2-IL15-CMV-tat (L1), pHi2-IL15-CEA-tat (L2), pHi2-spIL15-CMV-tat (L3) and pHi2-spIL15-CEA-tat (L4) by electroporation. Culture supernatant was collected $24 \mathrm{~h}$ following transfection, and IL-15 expression was detected by ELISA. The mean \pm SD of a representative of three triplicate experiments is shown. (B) Spleen cells were isolated and treated with the supernatant from IL-15 expression plasmid-transfected tumor cells. MTT assays were used to determine the proliferation of spleen cells. Supernatant from non-transfected tumor cells was used as a control. Data are representative of three triplicate experiments. ELISA, enzyme-linked immunosorbent assay; MTT, methyl thiazolyl tetrazolium.

contact to the skin. Pulses were applied to the tumor through the electrode using a power supply device (BTX ECM 830, Genetronic). The parameters were $200 \mathrm{~V} / \mathrm{cm}$, six $20-\mathrm{msec}$ pulses. Plasmid injection and electroporation were performed twice, on days 7 and 10. Tumors were measured every three days with calipers, and the tumor volume was calculated using the formula: length $\mathrm{x}$ width ${ }^{2} \mathrm{x} \pi / 6$.

Statistical analysis. Values are expressed as the means \pm standard deviations (SD). Results were compared using the Student's t-test. A p-value less than 0.05 was accepted as indicating a significant difference. Animal survival was evaluated by the Kaplan-Meier analysis.

\section{Results}

IL-15 expressionbytumorcellsfollowingelectroporationinvitro. SW480 and MCF-7 cells were transfected with plasmids pHi2IL15-CMV-tat, pHi-IL15-CEA-tat, pHi2-spIL15-CMV-tat and pHi2-spIL15-CEA-tat by electroporation. As previously described, plasmids pHi2-IL15-CMV-tat and pHi2-spIL15CMV-tat have the CMV promoter driving tat expression, whereas pHi2-IL15-CEA-tat and pHi-spIL15-CEA-tat have the CEA promoter driving tat expression. Plasmids pHi2IL15-CMV-tat and pHi2-IL15-CEA-tat have the natural IL-15 signal peptide, whereas pHi2-spIL15-CMV-tat and pHi-spIL15-CEA-tat have the IL-2 signal peptide, replacing the IL-15 signal peptide. As shown in Fig. 1A, pHi2-spIL15CMV-tat gave rise to the highest level of IL-15 expression in the two cell lines $(178.0 \mathrm{pg} / \mathrm{ml}$ for SW480 cells and $369.9 \mathrm{pg}$ / $\mathrm{ml}$ for MCF-7 cells). For the pHi2-spIL15-CEA-tat plasmid, a comparable level of IL-15 expression was achieved in CEA-positive SW480 cells (163.7 vs. $178.0 \mathrm{pg} / \mathrm{ml}, \mathrm{p}>0.05$ ), whereas in CEA-negative MCF-7 cells, a significantly lower IL-15 expression was detected (140.0 vs. $369.9 \mathrm{pg} / \mathrm{ml}$, $\mathrm{p}<0.05)$. Plasmids pHi2-IL15-CMV-tat and pHi2-IL15-CEAtat gave rise to lower levels of IL-15 expression compared to pHi2-spIL15-CMV-tat and pHi-spIL15-CEA-tat. These results confirmed that the IL-2 signal peptide was more efficient in causing IL-15 expression and that the CEA promoter was capable of inducing a targeted transgene expression in CEA-positive tumor cells. The transfection efficiency was $\sim 20 \%$ for SW480 cells and $26 \%$ for MCF-7 cells, as determined by transfection of the EGFP-expressing plasmid under the same conditions (data not shown).

IL-15 expressed by transfected tumor cells stimulated spleen cell proliferation in vitro. To determine the function of IL-15, spleen cells were cultured with the supernatant of tumor

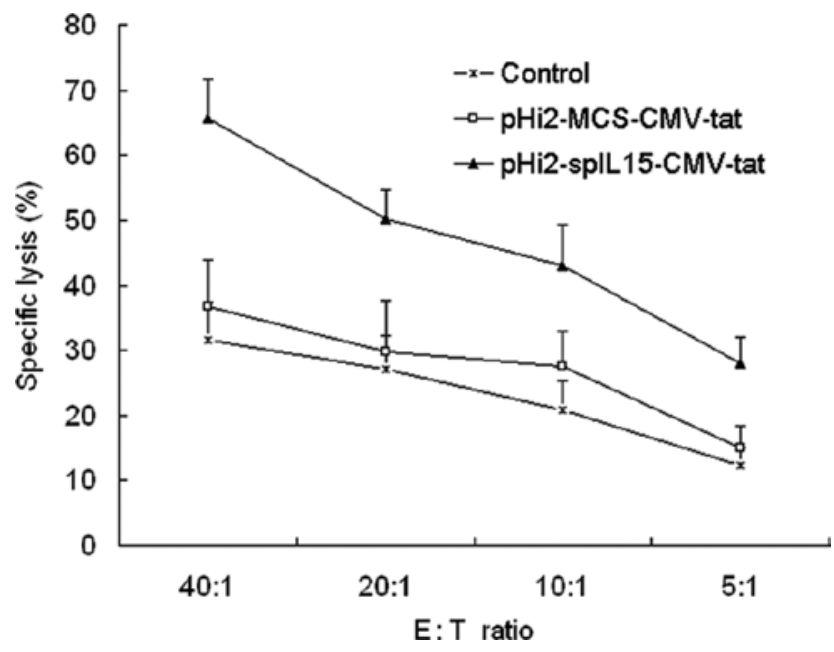

Figure 2. IL-15 gene transfer increased the cytotoxicity of NK cells. NK cells were isolated via negative selection using a MACS NK Cell Isolation Kit. Yac-1 cells were used as target cells. Cytotoxicity assays were performed using a CytoTox96 non-radioactive cytotoxicity assay kit. Specific lysis at four E:T ratios is shown. NK cells isolated from naïve mice were used as a control group. Data are representative of three triplicate experiments. 

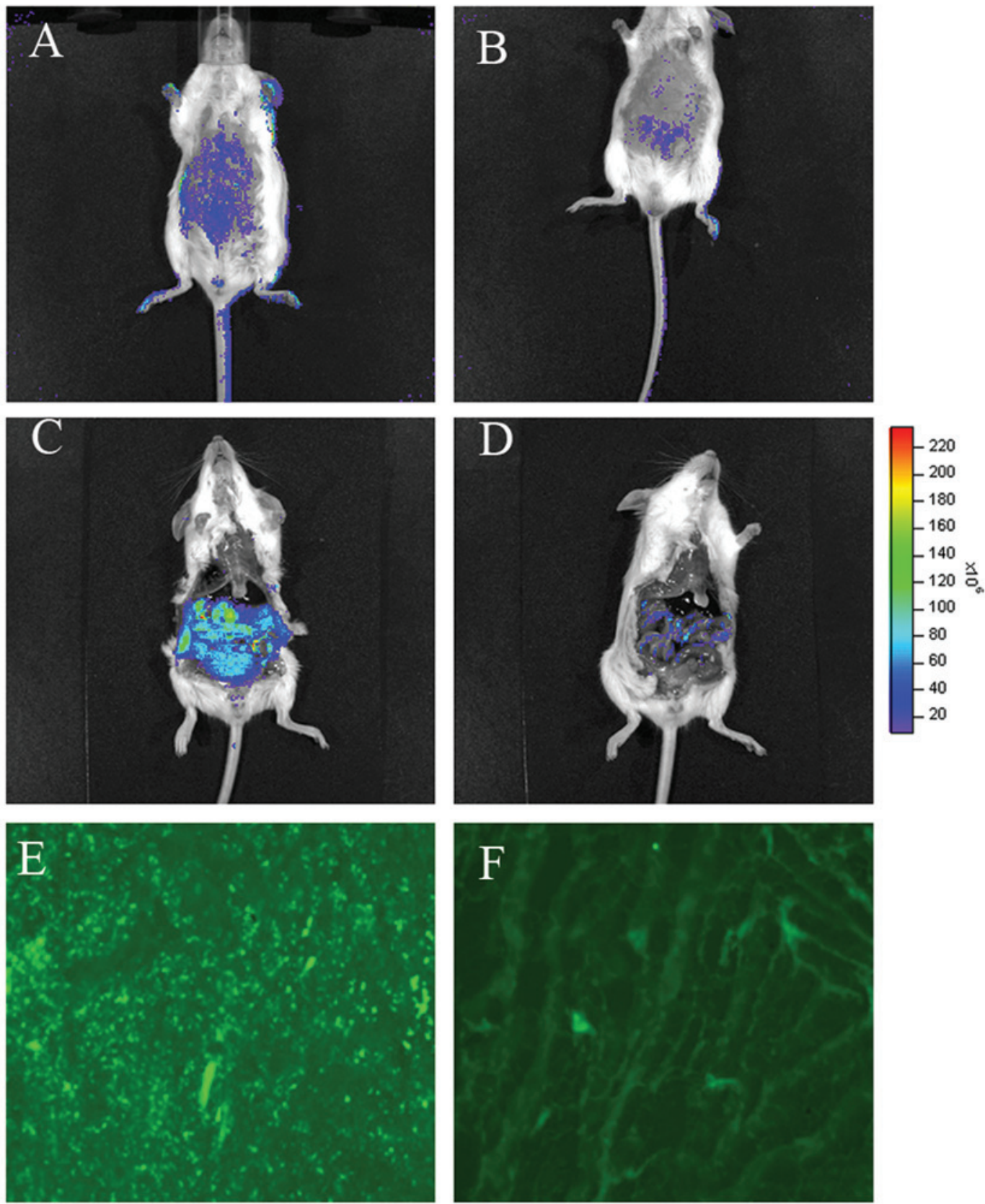

Figure 3. Gene expression of intraperitoneal and intra-tumor gene transfer. For intraperitoneal gene transfer, each mouse was injected intraperitoneally with $200 \mu \mathrm{l}$ of formulation containing (A and C) $50 \mu \mathrm{g} \mathrm{pHi}$-EGFP-CMV-tat plasmid DNA or (B and D) $200 \mu 1 \mathrm{PBS}, 24 \mathrm{~h}$ prior to imaging. Transgene expression was assessed by in vivo animal fluorescence and optical imaging was performed using the Xenogen system. (A) and (B) show whole-body imaging prior to opening the abdomen, and (C) and (D) show whole-body imaging after opening the abdomen. Images are representative of two experiments with 4 mice in each group. For intra-tumor gene transfer, the plasmid pHi2-EGFP-CMV-tat was injected into CT-26 tumors formed by the subcutaneous inoculation of CT-26 cells. Each mouse received (E) $50 \mu \mathrm{g}$ of naked plasmid or (F) PBS, followed by electroporation. Tumor tissue was harvested $24 \mathrm{~h}$ following gene transfer and was observed using a fluorescence microscope. Images are representative of two experiments with 4 mice in each group.

cells transfected with the IL-15 expression plasmids, and the proliferation of the spleen cells was monitored. Compared to the control medium, all supernatants harvested from the IL-15 expression plasmid-transfected SW480 cells, and supernatants harvested from pHi2-IL15-CMV-tat-, pHi2spIL15-CMV-tat- and pHi2-spIL15-CEA-tat-transfected MCF-7 cells, significantly stimulated spleen cell proliferation $(\mathrm{p}<0.05)$ (Fig. 1B). The stimulatory activity of supernatant harvested from pHi2-spIL16-CMV-tat-transfected SW480 cells resembled the activity of $125 \mathrm{pg} / \mathrm{ml}$ of recombinant IL-15 (absorbance value $0.690 \pm 0.100$ vs. $0.695 \pm 0.030$ ). These results indicate that IL-15 expressed by genetically modified tumor cells is biologically active and can stimulate spleen cell proliferation.

Enhanced cytotoxic activity of NK cells following IL-15 gene transfer. The cytotoxic activity of NK cells isolated from mice treated with IL-15-expressing plasmids was assessed using Yac-1 cells as target cells. BALB/c mice were injected intraperitoneally with IL-15-expressing plasmids or backbone plasmid on days 1, 4 and 7, and spleen cells were collected on day 10 . NK cells were isolated from the control mice, backbone 

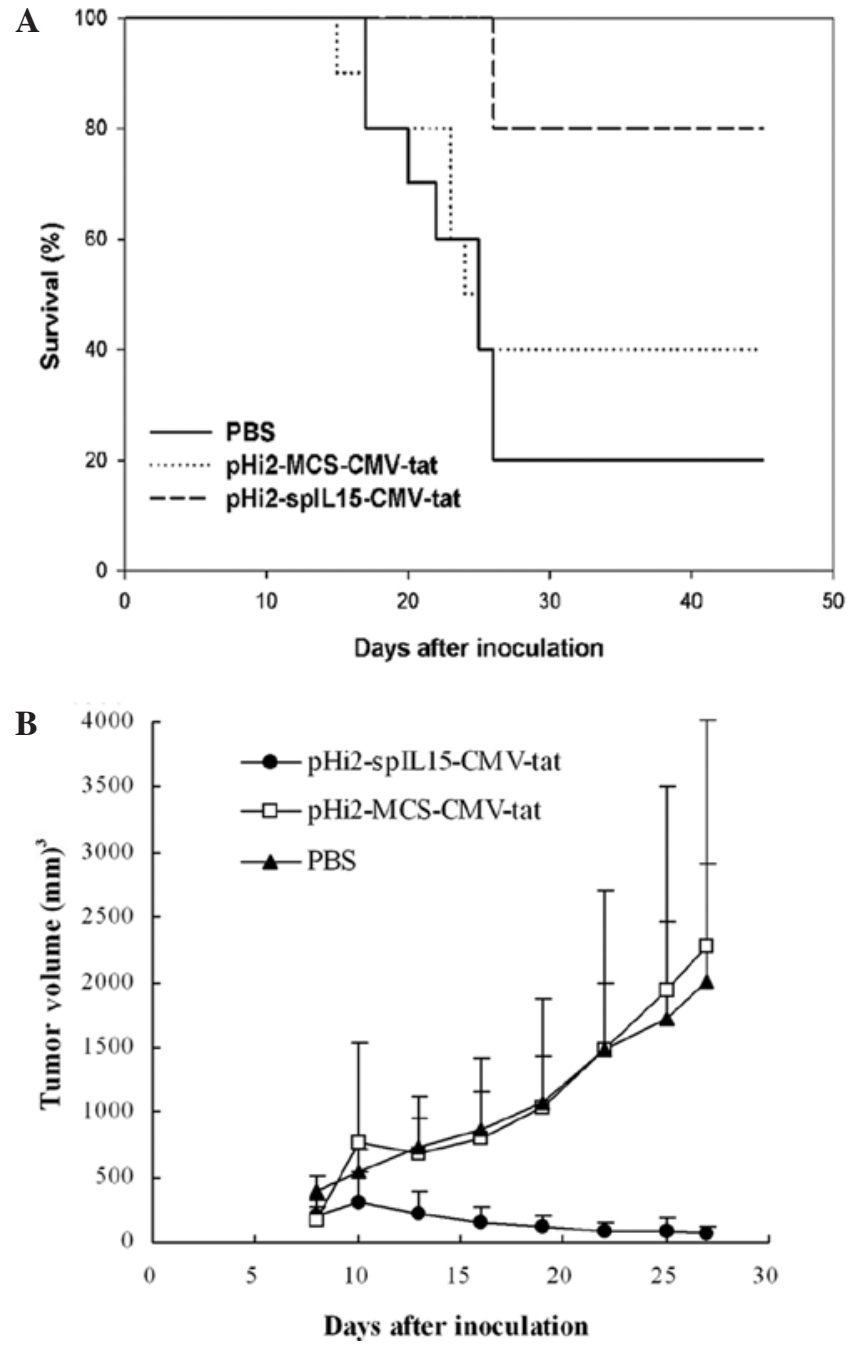

Figure 4. IL-15 plasmid injection increased the survival of mice inoculated with CT-26 tumor cells and decreased CT-26 tumor growth. (A) Mice were inoculated intraperitoneally with $1 \times 10^{7}$ CT-26 tumor cells and treated by abdominal injection with PBS (solid line), backbone plasmid pHi2-MCS-CMV-tat (dotted line), or IL-15 expression plasmid pHi2-spIL15-CMV-tat (dashed line) on days 3,5 and 7, and survival time was monitored. pHi2-spIL15-CMV-tat vs. PBS or pHi2-MCS-CMV-tat $(\mathrm{n}=10$ for each group), $\mathrm{P}<0.05$. Animal survival is shown using the Kaplan-Meier survival curves. (B) Mice were inoculated subcutaneously with $5 \times 10^{6} \mathrm{CT}-26$ tumor cells. After tumors were palpable at days 7 and 10, PBS (solid triangle), $50 \mu \mathrm{g}$ backbone plasmid pHi2-MCS-CMV-tat (square) or $50 \mu \mathrm{g} \mathrm{IL-15} \mathrm{expres-}$ sion plasmid pHi2-spIL15-CMV-tat (diamond) were electroporated into the tumor, and tumor volumes were monitored. Each value is the mean $\pm \mathrm{SD}$ ( $\mathrm{n}=6$ for each group). $\mathrm{pHi2}$-spIL15-CMV-tat vs. PBS or pHi2-MCS-CMVtat, $\mathrm{P}<0.05$. PBS, phosphate-buffered saline.

vector-treated mice and IL-15 expression plasmid-treated mice via negative selection using MACS beads. As shown in Fig. 2, NK cells isolated from all groups lysed Yac-1 cells efficiently. A significant increase in cytotoxicity was observed in the NK cells prepared from mice treated with IL-15-expressing plasmids. At the effector to target cell ratio of $40: 1$, the specific lysis of NK cells prepared from pHi2-spIL15-CMV-tat-treated mice was $65.6 \%$, whereas the specific lysis of NK cells prepared from control and pHi2-MCS-CMV-tat-treated mice was 31.6 and $36.7 \%$, respectively $(\mathrm{p}<0.05)$.

Transgene expression in the peritoneum followed intraperitoneal injection of naked DNA plasmid or in vivo electroporation. To determine whether intraperitoneal plasmid injection is capable of inducing efficient transgene expression, EGFP-expressing plasmid pHi2-EGFP-CMV-tat was injected into the peritoneal cavity of BALB/c mice. Each mouse was injected with $200 \mu \mathrm{l}$ of formulation containing $50 \mu \mathrm{g}$ plasmid DNA or $200 \mu \mathrm{l}$ PBS $24 \mathrm{~h}$ prior to imaging. Transgene expression was assessed by in vivo animal fluorescence optical imaging using the Xenogen system (Xenogen). GFP expression was observed in the plasmid-treated mice (Fig. 3 A-D), indicating that the peritoneal mesothelial cells acquired the plasmids and that the transgene was expressed. In addition, to assess in vivo transgene expression in tumor tissue following electroporation, the pHi2-EGFP-CMV-tat plasmid was injected into tumors formed by the subcutaneous inoculation of CT-26 cells. Each mouse received $50 \mu \mathrm{g}$ of naked plasmid or PBS, followed by electroporation. Tumor tissue was harvested $24 \mathrm{~h}$ following gene transfer and observed using a fluorescence microscope. GFP expression was observed in pHi2-GFP-CMV-tat-treated tumor tissue (Fig. 3E and F).

Decreased intraperitoneal and subcutaneous tumor formation following $I L-15$ expression plasmid gene transfer. The therapeutic effect of IL-15-expressing plasmids was assessed using an intraperitoneal tumor formation model or a subcutaneous tumor formation model with CT-26 cells in BALB/c mice. We monitored the mice for 45 days following intraperitoneal inoculation of the CT-26 cells. Most mice in the control groups died in the fourth week post-inoculation; however, $80 \%$ of the mice treated with IL-15 expression plasmids survived, and there was no sign of tumor growth in these animals during the observation period (Fig. 4A). These data indicate that an intraperitoneal injection of the IL-15 expression plasmid pHi2-spIL15-CMV-tat is capable of increasing the survival of mice inoculated intraperitoneally with CT-26 tumor cells. Similarly, electroporation of pHi2-spIL15-CMV-tat also inhibited the formation of tumors in mice subcutaneously injected with CT-26 cells. Compared to the PBS control and backbone vector groups, all of the tumors stopped growing in mice that received three treatments of electroporation with $50 \mu \mathrm{g}$ pHi2-spIL15-CMV-tat plasmid (Fig. 4B). Mice were monitored for a total of 45 days and revealed no signs of tumor recurrence.

\section{Discussion}

Cytokines play an essential role in the regulation of immune responses and hold great promise for cancer immunotherapy. IL-15, with its broad activity on cells involved both in innate and adaptive immunity, is an attractive candidate for immunogene therapy. IL-15 was first isolated from a simian kidney epithelial cell line in 1994 by Grabstein et al (7). It promotes the survival, proliferation, activation and maintenance of $\mathrm{NK}$ and $\mathrm{CD} 8^{+} \mathrm{T}$ cells, and it also stimulates the function of neutrophils, macrophages and dendritic cells. Overexpression of IL-15 has been shown to result in the expansion and activation of $\mathrm{NK}$ cells, CD44 ${ }^{\text {(hi) }} \mathrm{CD} 8+$ +memory cells and $\gamma \delta \mathrm{T}$ cells (8). IL-15 contributes to enhanced immune memory by selectively propagating memory $\mathrm{T}$ cells and by blocking IL-2mediated T-cell death (9). IL-15 has also been shown to be a chemoattractant for T lymphocytes, NK cells and neutrophils. 
In addition, IL-15 is a potent inducer of CC-, CXC- and C-type chemokine production in T lymphocytes (10).

The administration of IL-15 has been shown to effectively inhibit tumor growth in mice (11). Enhanced tumor immunosurveillance has been demonstrated in IL-15 transgenic mice (12). Using IL-15 transgenic mice, Yajima et al (13) examined the effects of in vivo IL-15 overexpression on protection against murine B16 melanoma, and they demonstrated that tumor growth was severely retarded in transgenic mice following subcutaneous inoculation with B16.44 or B16F10 cells. IL-15 transgenic mice revealed enhanced antitumor activities of NK cells and CD $8^{+} \mathrm{T}$ cells (13). In a previous study, we constructed the amplified IL-15-expressing plasmid vector, pHi2-spIL15-CMV-tat, to achieve a high level of IL-15 expression (6). In the present study, we applied our IL-15 expression vector to a clinically relevant technique. We demonstrated that intraperitoneal gene transfer and electroporation of plasmid pHi-spIL15-CMV-tat protected mice from CT-26 tumor challenge.

The peritoneal cavity is a common site for colon and gastrointestinal cancer metastasis and recurrence following surgical treatment. There are abundant mesothelial cells in the peritoneal cavity. Previous studies have indicated that peritoneal mesothelial cells have a potent ability to produce a number of cytokines (14). Tanaka et al reported that IL-10 expressed by peritoneal mesothelial cells via gene transfer was capable of suppressing the peritoneal dissemination of malignant tumor cells (15). In this study, we investigated whether the expression of IL-15 within the peritoneal cavity was capable of enhancing the ability of immune cells to control tumor growth. We demonstrated that the intraperitoneal injection of our plasmid vector resulted in a significant amount of transgene expression by mesothelial cells and, importantly, that intraperitoneal IL-15 gene transfer decreased the growth of CT-26 tumors. Naked DNA has been shown to be a safe, simple and inexpensive approach for gene delivery. Therefore, intraperitoneal IL-15 gene therapy may be used in clinical settings to control peritoneal carcinomatosis caused by tumor recurrence following surgery.

To investigate whether the expression of IL-15 in the tumor tissue would result in a biological effect, we transfected a subcutaneously grown tumor with IL-15 plasmid via electroporation. Electroporation is one of the most commonly used methods for in vivo gene transfer, and numerous cytokines have been investigated for cancer immunogene therapy using electroporation, including IL-2, IL-12 and GM-CSF (16-18). Electroporation has also been applied in clinical trials; Daud et al (19) reported that IL-12 plasmid electroporation in patients with metastatic melanoma resulted in increases of IL-12 protein levels proportional to the plasmid dose, as well as marked tumor necrosis and lymphocytic infiltration. A total of $42 \%$ of patients in that study exhibited disease stabilization or a partial response (19). In the present study, we have demonstrated that intratumoral IL-15 gene transfer by electroporation is capable of decreasing tumor growth.

In conclusion, the IL-15-expressing plasmids we constructed achieved efficient transgene expression following in vitro and in vivo gene transfer. Intraperitoneal injection of plasmids results in transgene expression by epithelial cells in the peritoneum. An IL-15-expressing plasmid is capable of decreasing the growth of inoculated tumor cells following intraperitoneal injection and is capable of prolonging mouse survival. Electroporation of the IL-15-expressing plasmid into tumor tissue may retard the growth of tumors in mice subcutaneously inoculated with CT-16 cancer cells. Therefore, this study provides evidence for the application of IL-15 in cancer gene therapy and for the continued examination of amplified IL-15 expression plasmid vectors for use in preclinical settings.

\section{Acknowledgements}

The project was sponsored by the Scientific Research Foundation for the Returned Overseas Chinese Scholars, State Education Ministry (to Dr Xianghui He), Research Foundation of Tianjin Medical University (to Dr Xianghui He).

\section{References}

1. Jinushi $\mathrm{M}$ and Tahara H: Cytokine gene-mediated immunotherapy: current status and future perspectives. Cancer Sci 100: 1389-1396, 2009.

2. Edelstein ML, Abedi MR and Wixon J: Gene therapy clinical trials worldwide to 2007 - an update. J Gene Med 9: 833-842, 2007.

3. Kennedy MK, Glaccum M, Brown SN, Butz EA, Viney JL, Embers M, Matsuki N, Charrier K, Sedger L, Willis CR, Brasel K, Morrissey PJ, Stocking K, Schuh JC, Joyce S and Peschon JJ: Reversible defects in natural killer and memory CD8 $\mathrm{T}$ cell lineages in interleukin 15-deficient mice. J Exp Med 191: 771-780, 2000.

4. Teague RM, Sather BD, Sacks JA, Huang MZ, Dossett ML, Morimoto J, Tan X, Sutton SE, Cooke MP, Ohlen C and Greenberg PD: Interleukin-15 rescues tolerant CD8+ $\mathrm{T}$ cells for use in adoptive immunotherapy of established tumors. Nat Med 12: 335-341, 2006.

5. Mortier E, Woo T, Advincula R, Gozalo S and Ma A: IL-15Ralpha chaperones IL-15 to stable dendritic cell membrane complexes that activate NK cells via trans presentation. J Exp Med 205: 1213-1225, 2008.

6. He X, Li W, Lu N, Qi F, Zhao Na, Qiu Y and Zhu L: Amplified interleukin-15 expression vectors for cancer immunogene therapy. Mol Med Report 1: 369-374, 2008.

7. Grabstein KH, Eisenman J, Shanebeck K, Rauch C, Srinivasan S Fung V, Beers C, Richardson J, Schoenborn MA, Ahdieh M, et al: Cloning of a T cell growth factor that interacts with the beta chain of the interleukin-2 receptor. Science 264: 965-968, 1994.

8. Dubois S, Patel HJ, Zhang M, Waldmann TA and Muller JR: Preassociation of IL-15 with IL-15R alpha-IgG1-Fc enhances its activity on proliferation of NK and CD8+/CD44high T cells and its antitumor action. J Immunol 180: 2099-2106, 2008.

9. Marks-Konczalik J, Dubois S, Losi JM, Sabzevari H, Yamada N, Feigenbaum L, Waldmann TA and Tagaya Y: IL-2-induced activation-induced cell death is inhibited in IL-15 transgenic mice. Proc Natl Acad Sci USA 97: 11445-11450, 2000.

10. Perera LP, Goldman CK and Waldmann TA: IL-15 induces the expression of chemokines and their receptors in $\mathrm{T}$ lymphocytes. J Immunol 162: 2606-2612, 1999.

11. Evans R, Fuller JA, Christianson G, Krupke DM and Troutt AB: IL-15 mediates anti-tumor effects after cyclophosphamide injection of tumor-bearing mice and enhances adoptive immunotherapy: the potential role of NK cell subpopulations. Cell Immunol 179: 66-73, 1997.

12. Kobayashi H, Dubois S, Sato N, Sabzevari H, Sakai Y, Waldmann TA and Tagaya Y: Role of trans-cellular IL-15 presentation in the activation of NK cell-mediated killing, which leads to enhanced tumor immunosurveillance. Blood 105: 721-727, 2005.

13. Yajima T, Nishimura H, Wajjwalku W, Harada M, Kuwano H and Yoshikai Y: Overexpression of interleukin-15 in vivo enhances antitumor activity against MHC class I-negative and -positive malignant melanoma through augmented $\mathrm{NK}$ activity and cytotoxic T-cell response. Int J Cancer 99: 573-578, 2002. 
14. Lanfrancone L, Boraschi D, Ghiara P, Falini B, Grignani F, Peri G Mantovani A and Pelicci PG: Human peritoneal mesothelial cells produce many cytokines (granulocyte colony-stimulating factor [CSF], granulocyte-monocyte-CSF, macrophage-CSF interleukin-1 [IL-1], and IL-6) and are activated and stimulated to grow by IL-1. Blood 80: 2835-2842, 1992.

15. Tanaka F, Tominaga K, Shiota M, Ochi M, Kuwamura H, Tanigawa T, Watanabe T, Fujiwara Y, Oshitani N, Higuchi K, Iwao $\mathrm{H}$ and Arakawa T: Interleukin-10 gene transfer to peritoneal mesothelial cells suppresses peritoneal dissemination of gastric cancer cells due to a persistently high concentration in the peritoneal cavity. Cancer Gene Ther 15: 51-59, 2008.

16. Heller L, Pottinger C, Jaroszeski MJ, Gilbert R and Heller R: In vivo electroporation of plasmids encoding GM-CSF or interleukin-2 into existing B16 melanomas combined with electrochemotherapy induces long-term antitumour immunity. Melanoma Res 10: 577-583, 2000.
17. Goto T, Nishi T, Kobayashi O, Tamura T, Dev SB, Takeshima H, Kochi M, Kuratsu J, Sakata T and Ushio Y: Combination electro-gene therapy using herpes virus thymidine kinase and interleukin-12 expression plasmids is highly efficient against murine carcinomas in vivo. Mol Ther 10: 929-937, 2004.

18. Tangney M,Casey G, Larkin JO, Collins CG, Soden D, Cashman J, Whelan MC and O'Sullivan GC: Non-viral in vivo immune gene therapy of cancer: combined strategies for treatment of systemic disease. Cancer Immunol Immunother 55: 1443-1450, 2006.

19. Daud AI, DeConti RC, Andrews S, Urbas P, Riker AI, Sondak VK, Munster PN, Sullivan DM, Ugen KE, Messina JL and Heller R: Phase I trial of interleukin-12 plasmid electroporation in patients with metastatic melanoma. J Clin Oncol 26: 5896-5903, 2008. 